\title{
FORMULATION AND EVALUATION OF DEFLAZACORT LOADED GELATIN MICROSPHERES
}

\author{
Daisy Chellakumari*1, A. Selvapriya ${ }^{1}$, C. Soniya ${ }^{2}$ and A. Dinesh Kumar ${ }^{1}$ \\ ${ }^{1}$ College of Pharmacy, Madras Medical College. \\ ${ }^{2}$ College of Pharmacy, Madurai Medical College.
}

\begin{abstract}
In the present study formulation and characteristic of microspheres of Deflazacort using gelatin in a sustained release form, to overcome drug resistance and dosing non-compliance in patients. Deflazacort is used as an antiinflammatory belonging to a systemic corticosteroid and it was successfully encapsulated into carrier polymer gelatin and cross- linked with glutaraldehyde. The effect of gelatin concentration and cross-linking agent was evaluated with respect to surface characteristic particle size, entrapment efficiency, micromeritics study and in vitro release study. Infrared spectroscopic analysis confirmed the absence of any drug polymer interaction. Drug entrapment efficiency for deflazacort F4 formulation reached to highest level of $86.5 \%$ and percentage yield to 89.0\%. The formulated microspheres gave $84.94 \%$ drug release in a sustained manner for $12 \mathrm{~h}$. This gave hope for the possibility of single dose treatment for patients. The kinetics analysis of the drug release from the gelatin microspheres follows first order and anomalous transport mechanism.
\end{abstract}

\section{KEY WORDS}

Deflazacort, Gelatin, Glutaraldehyde, Microspheres

\section{INTRODUCTION}

Oral controlled release dosage forms have been developed over the past three decades due to their considerable therapeutic advantages such as ease of administration, patient compliance and flexibility in formulation. Microsphere carrier systems made from the naturally occurring biodegradable polymers have attracted considerable attention for several years in sustained drug delivery. Recently, dosage forms that can precisely control the release rates and target drugs to a specific body site have made an enormous impact in the formulation and development of novel drug delivery systems ${ }^{1-3}$. They have varied applications and are prepared using assorted polymers ${ }^{4}$. However, the success of these microspheres is limited owing to their short residence time at the site of absorption. It would, therefore, be advantageous to have means for providing an intimate contact of the drug delivery system with the absorbing membranes ${ }^{5-8}$. This can be achieved by coupling bioadhesion characteristics to microspheres and developing bioadhesive microspheres. Bioadhesive microspheres have advantages such as efficient absorption and enhanced bioavailability of drugs owing to a high surface-tovolume ratio, a much more intimate contact with the mucus layer, and specific targeting of drugs to the absorption site ${ }^{9-12}$. Gelatin (obtained by boiling skin, tendons and ligaments with water) is a cationic polymer that has been proposed for use in microsphere systems by a number of authors ${ }^{13-17}$. Gelatin was selected as a polymer in the preparation of mucoadhesive microspheres because of its good mucoadhesive and biodegradable properties. Hence, there is a need to develop an oral drug delivery system that is, convenient for patients. Various synthetic and natural polymers like alginate, gelatin and chitosan have been used to develop drug delivery systems for entrapping and delivery drugs orally ${ }^{18}$. 


\section{MATERIALS AND METHODS}

Deflazacort was collected as a gift sample from Alcon bio sciences PVT Ltd, Mumbai. Gelatin was collected as a gift sample from Bafna Pharma, Chennai. Glutaraldehyde and liquid paraffin was purchased from SD fine chemicals PVT Ltd, Mumbai and all other reagents were of analytical grade.

Preparation of Deflazacort microspheres by using gelatin polymer by Emulsification method

The Gelatin microspheres were prepared by an Emulsification technique by some modifications of MuniyandySaravanan ${ }^{19}$ et al.10ml of $15 \%(\mathrm{w} / \mathrm{v})$ aqueous gelatin solution was prepared and preheated to $60^{\circ} \mathrm{C}$. Deflazacort was dissolved in ethanol and added to gelatin solution. Then this mixture was added drop wise to $50 \mathrm{ml}$ of light liquid paraffin containing $1 \% \mathrm{w} / \mathrm{v}$ span 80 preheated to $60^{\circ} \mathrm{C}$ and the dispersion was stirred continuous for $20 \mathrm{~min}$ using magnetic stirrer at 1000 rpm. Then $0.1 \mathrm{ml}$ of cross linking agent glutaraldehyde saturated with toluene solution was added and stirring was continued for $3 \mathrm{~h}$. The microspheres were centrifuged, washed several times with hexane and finally with acetone. The microspheres were then dried at room temperature and stored in desiccator. Five different formulations with drug: polymer ratios (1:1, $1: 2,1: 3,1: 4,1: 5$ ) were prepared and coded as F1, F2, F3, F4 and F5.

\section{Evaluation of Microspheres}

\section{Drug Polymer interaction (FTIR) Study ${ }^{20}$}

IR spectroscopy was performed on Fourier transformer infrared spectrometer (Nicolet, India). The pellets of drug and potassium bromide (1:1) were prepared by compressing at 20 psi for 10 min on $\mathrm{KBr}$-press and the spectra were scanned in the wave number range of $4000-600 \mathrm{~cm}^{-1}$ for the sample of Deflazacort, Gelatin and Physical mixture of drug and polymer.

\section{Particle size analysis ${ }^{21}$}

The samples of the optimized formulation (F4), were analyzed for their particle size distribution by using Malvern Particle Size Analyzer.

Percentage yield ${ }^{21}$

The Percentage yield of prepared microspheres was de termined by using the formula.

Percentage yield $=\frac{\text { Practical yield }}{\text { Theoritica lyield }} \times 100$

\section{Determination of drug content}

$100 \mathrm{mg}$ of microspheres of F1, F2, F3, F4 and F5was weighed and dissolved in $10 \mathrm{ml}$ of ethanol. The solution was suitably diluted with phosphate buffer $\mathrm{pH} 7.4$ in $100 \mathrm{ml}$ standard flask and filtered to separate the fragments, and drug content was analysed by UV spectrophotometer (SHIMADZU, Japan) at a wavelength of $247 \mathrm{~nm}$.

\section{Determination of entrapment efficiency ${ }^{21}$}

The entrapment efficiency was calculated from the ratio of actual to theoretical drug content and expressed as percentage. The formula applied is

Percentage drug entrapment efficiency $=\frac{\text { Actual drug content }}{\text { Theoritical drug content }}$ $\times 100$

Characterization of flow properties ${ }^{22,23,24}$

Flow properties of microspheres were investigated by determining by following standard procedures.

\section{Bulk Density}

Bulk density was determined by taking a known weight of dried microspheres in a measuring cylinder and tapping 3 times from 1 inch height at 2 sec. interval. The Bulk volume is noted and the Bulk density was calculated from the following equation.

\section{Bulk density $=\frac{\text { Weight of microspheres }}{\text { Bulk volume of microspheres }}$ Tapped Density}

Tapped density is the ratio of mass of microsphere to the volume occupied by the same mass of the powder after a standard tapping of a measure. Weighed quantity of microspheres was taken in a cylinder and tapping 300 times from 1 inch at 2 sec intervals. The tapped volume is noted and the tapped density was calculated from the equation

Tapped density $=\frac{\text { Weight of microspheres }}{\text { Tapped volume of microspheres }}$

\section{Hausner's Ratio}

Hausner's ratio is used for predicting the flow characteristics

$$
\text { Hausner's ratio }=\frac{\text { Tapped density }}{\text { Bulk density }}
$$




\section{Compressibility Index}

Compressibility index was determined by using bulk density and tapped density.

Compressibilty index $=\frac{\text { Tapped density-Bulk density }}{\text { Tapped density }} \times 100$

\section{Angle of Repose}

A funnel was fixed to a stand and bottom of the funnel was fixed at a height of $3 \mathrm{~cm}$ from the plane. Microspheres were placed in funnel and allowed to flow freely and the height and radius of the heap of microspheres was measured.

$$
\theta=\tan ^{-1} h / r
$$

Where, ' $h$ ' is the height of heap and

' $r$ ' is the radius of heap of microspheres.

\section{In vitro drug release ${ }^{25}$}

In vitro drug release study was carried out in USP XXIII basket type dissolution test apparatus using phosphate buffer pH7.4 as dissolution medium, Volume of dissolution medium was $900 \mathrm{ml}$ and bath temperature was maintained at $(37 \pm 1)^{\circ} \mathrm{C}$ throughout study. Stirring speed was adjusted to $100 \mathrm{rpm}^{26}$. At predetermined time intervals, an aliquot of $5 \mathrm{ml}$ was withdrawn and replenished with fresh medium. Amount of drug in each aliquot was determined using a UV-Spectrophotometer at $247 \mathrm{~nm}$

\section{RESULTS AND DISCUSSION}

In the present work sustained release microspheres of deflazacort were formulated using gelatin polymer and glutaraldehyde as a cross-linking agent. Five different formulations with Drug: Polymer ratios $(1: 1,1: 2,1: 3,1: 4,1: 5)$ were prepared and evaluated for FTIR, Particle size distribution, Percentage yield, Percentage drug content, Entrapment efficiency, In vitro drug release and Kinetic analysis. The Results are shown in Table. 1

The FTIR spectra of Deflazacort, Gelatin, Physical mixture of Deflazacort and Gelatin and Optimized microsphere formulation F4 were shown in the Fig 1. From this it is clear that the peaks at $\mathrm{O}-\mathrm{H}$ stretch (3450.0), aromatic $\mathrm{C}-\mathrm{H}$ stretch (3070.0), aliphatic $\mathrm{C}-\mathrm{H}$ stretch (2935.0), C=O stretch (1740), aromatic C=C stretch (1650) and aromatic C-C stretch (1245.1) $\mathrm{cm}^{-1}$ are present in both the pure and formulations without any change in their positions indicating no chemical interaction between Deflazacort and Polymers. The sustained release microspheres of Deflazacort prepared by cross-linking were found to be almost spherical and free flowing.

The particle size analysis by Malvern sizer reveals that the average mean diameter of F4 formulation was found to be $1178(1.18 \mu) \mathrm{nm}$ (Fig 2).

It was observed that as the polymer ratio in the formulation increase, the product yield also increases. The low Percentage yielding some formulation may be due to microspheres lost during the washing process. The Percentage drug content in the formulation was found to be $32.94 \%$ to $66.50 \%$. Entrapment efficiency of microspheres was found to be $42.56 \%$ to $86.50 \%$. The Percentage Drug Entrapment efficiency of all batches varied. The idea of Percentage of loading and dosage calculation is obtained from the Percentage drug entrapment efficiency data. As the drug entrapment efficiency is nearer to $100 \%$ for any batch it shows best drug loading and required less amount of formulation dosage to be administered, compared to the less percentage drug entrapment batch. Here F4 gave highest drug entrapment efficiency and selected as $s$ best formulation for further analysis.

The In vitro performance of Deflazacort microspheres showed prolonged and sustained release of Deflazacort. The results of the In vitro dissolution studies of formulation F1 to F5 are showing Fig 3. Deflazacort release from the microsphere was studied for $12 \mathrm{~h}$ the drug released at constant rate in all the preparation and showed sustained release. The results of F4 formulation prepared with 1:4 concentration of drug: gelatin showed a release of maximum $84.94 \%$ at $\mathrm{pH} 7.4$ in $12 \mathrm{~h}$, similar pattern was observed in the case of other formulations. Data obtained from in vitro release studies was utilized for release kinetics. The in vitro release of $\mathrm{F} 4$ was fit into various kinetic models to find out the mechanism of drug release. Among this highest correlation coefficient was shown in higuchi release kinetics ( $r^{2}=0.893$ ) followed by first order $\left(r^{2}=0.824\right)$. The slope of the koresmeyer peppas plot ( $n=0.6305$ ) was found to be more than 5 indicating the diffusion was anomalous diffusion (non fickian diffusion). 
Table 1: Percentage yield, Drug content and Encapsulation efficiency of Deflazacort Microspheres

\begin{tabular}{lllll}
\hline Batch code & Drug: Polymer & Yield (\%) & Drug content (\%) & Encapsulation efficiency (\%) \\
\hline F1 & $1: 1$ & 63.20 & 32.94 & 42.56 \\
F2 & $1: 2$ & 65.20 & 38.49 & 46.56 \\
F3 & $1: 3$ & 66.50 & 41.34 & 51.20 \\
F4 & $1: 4$ & 67.00 & 66.50 & 86.50 \\
F5 & $1: 5$ & 68.90 & 38.50 & 48.40 \\
\hline
\end{tabular}

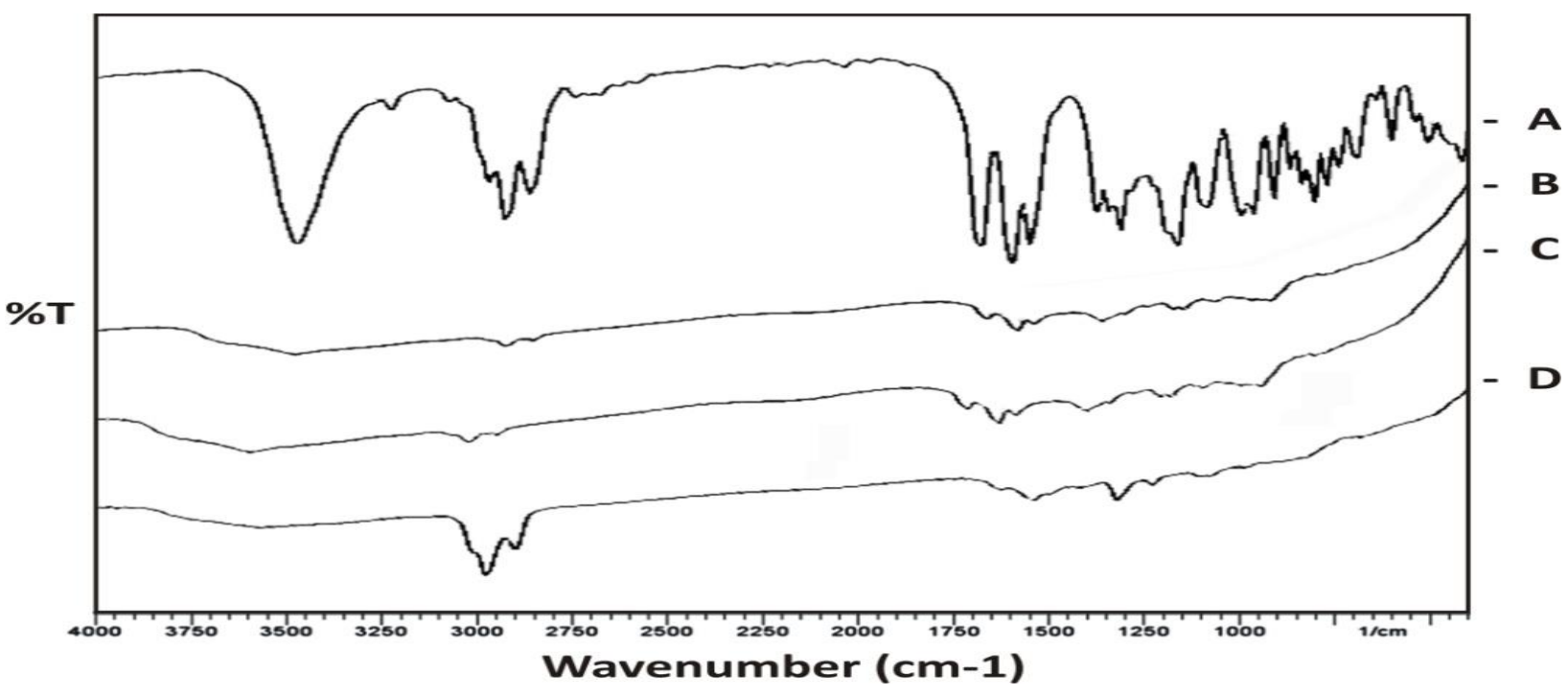

Fig. 1: Fourier transform infrared (FTIR) spectrum

A- Deflazacort, B- Gelatin, C-Physical mixture of Deflazacort and Gelatin, D-Microsphere formulation F4

$\begin{array}{rlllll} & & \text { Size (d.n... } & \text { \% Intensity: } & \text { St Dev (d.n... } \\ \text { Z-Average (d.nm): } & 1178 & \text { Peak 1: } & 849.7 & 97.6 & 153.7 \\ \text { Pdl: } & 0.494 & \text { Peak 2: } & 5560 & 2.4 & 6.104 \mathrm{e}-5 \\ \text { Intercept: } & 0.774 & \text { Peak 3: } & 0.000 & 0.0 & 0.000\end{array}$

Result quality Refer to quality report

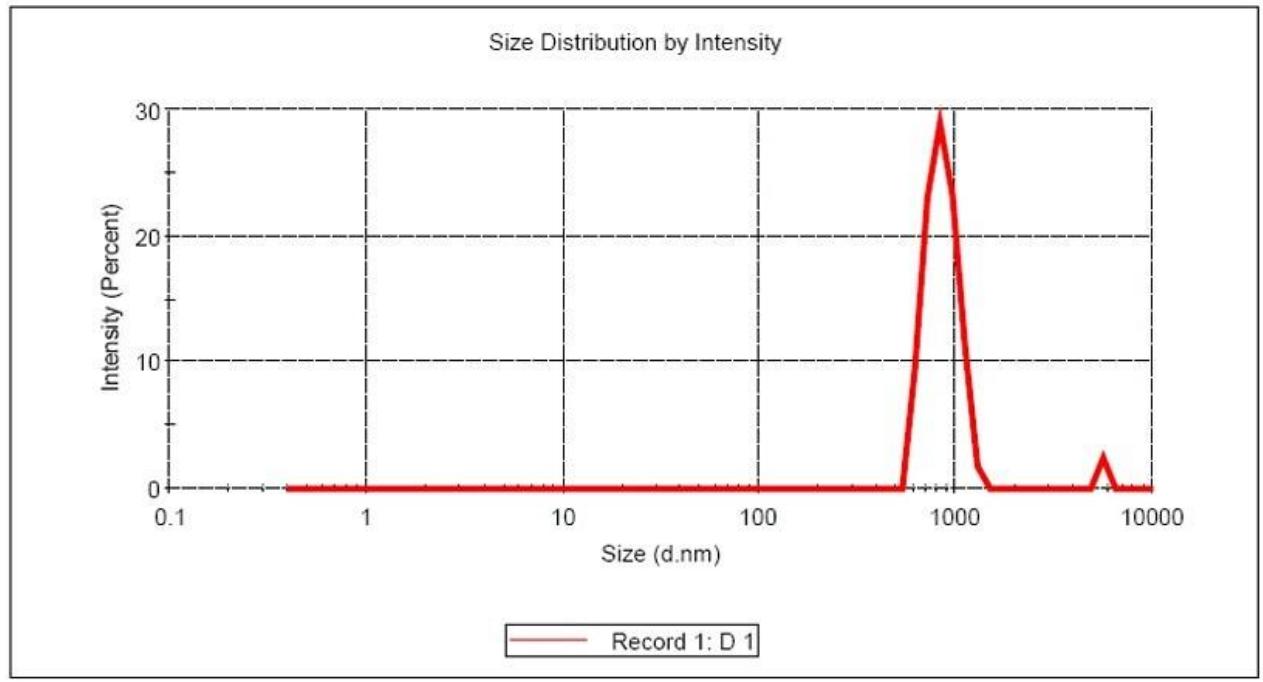

Fig.2: Particle size distribution of Deflazacort Microspheres 


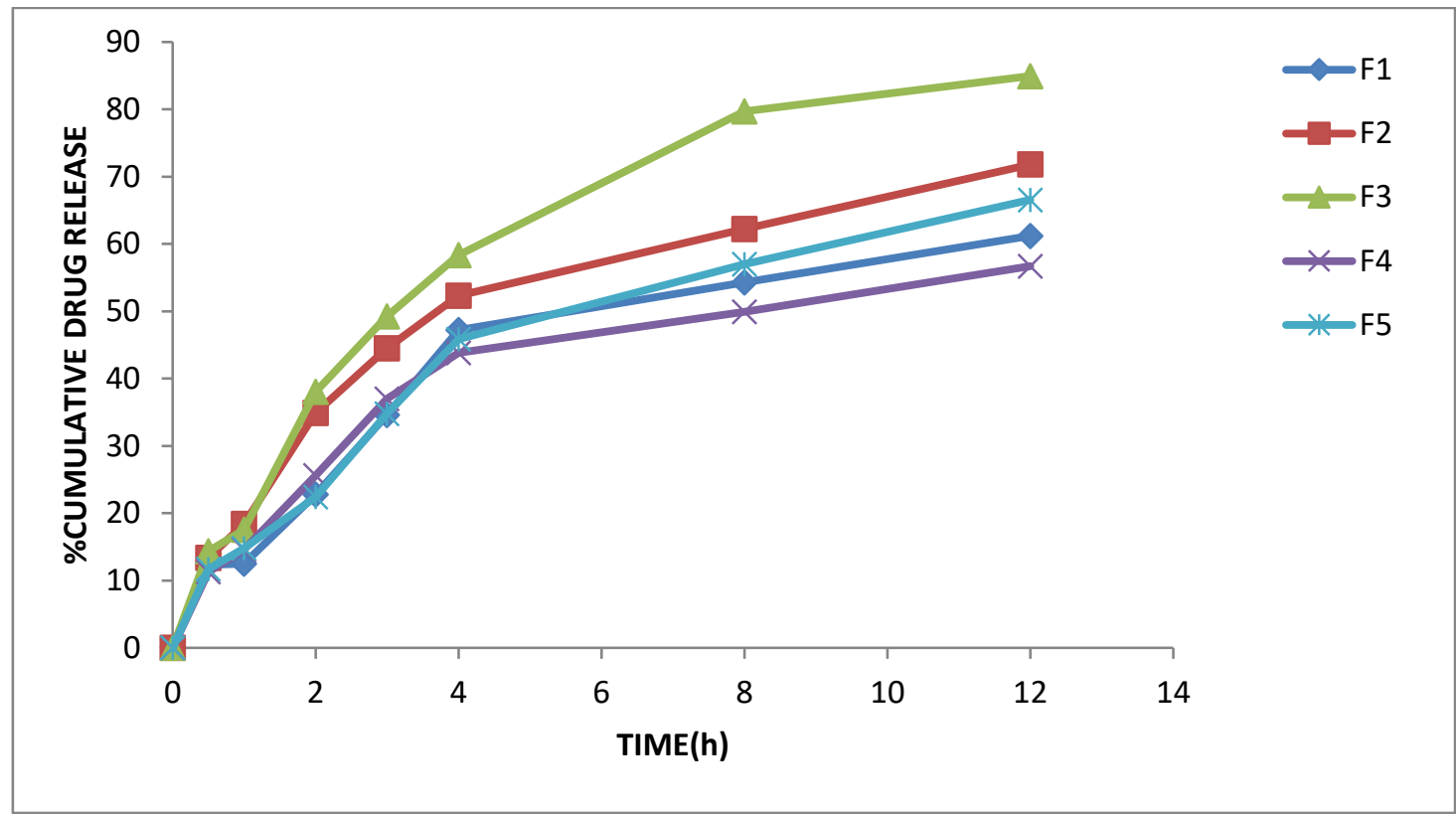

Fig.3: In vitro release of Deflazacort microspheres

\section{CONCLUSION}

The present study demonstrated the successful formulation of Deflazacort microsphere using gelatin as natural biodegradable polymer and release the drug in a sustained manner for an extended period of time. This can help to maximize the therapeutic effect of the drug, reduce side effect, decrease the dose and frequency of dosing and also helps to improve patient compliance.

\section{ACKNOWLEDGEMENT}

Authors are thankful to College of Pharmacy, Madras Medical College, for their support encouragement in carrying out this work.

\section{REFERENCES}

1. Woo BH, Jiang G, Jo YW, Deluca PP. Preparation and characterisation of a composite PLGA and poly (acryloylhydroxyl methyl starch) microsphere system for protein delivery. Pharm Res, 18:1600-1606, (2001)

2. Capan Y, Jiang G, Giovagnoli S, Deluca PP. Preparation and characterization of Poly (D, L-lactide -co-glycolide ) microspheres for controlled release of human growth hormone. AAPS Pharm Sci Tech, 4: E28,(2003)

3. Gohel MC, Amin AF. Formulation and optimization of controlled release Diclofenac sodium microspheres using factorial design. J control release. 51:115-122, (1998).
4. Vasir JK, Tamwekar K, Garg S. Bioadhesive microspheres as a controlled drug delivery system. Int J Pharm, 255:13-32, (2003).

5. Ikeda K, Murata K, Kobayashi M, Noda K. Enhancement of bioavailability of Dopaminevia nasal route in beagle dogs. Chem. Pharm Bull (Tokyo),40:2155-2158, (1992)

6. Nagai T, Nishimoto $Y$, Nambuu N, Suzuki Y, Sekine K. Powder dosage form of insulin for nasal administration . J Control Release, 1:15-22,(1984)

7. Ilium L, Farraj NF, Critchley H, Davis SS. Nasal administration of gentamicin using a novel microsphere delivery system .Int J Pharm ,46:261165,(1998)

8. Schaefer MJ, Singh J. Effect of isopropyl myristic acid ester on the physical characteristics and In vitro release of etoposide from PLGA microspheres. AAPS Pharm Sci Tech,1:E32,(2000)

9. Rao SB, Sharma CP. Use of gelatin as Biomaterial: studies on its safety and hemostatic potential. J Biomed Mater Res, 34:21-28, (1997)

10. Lehr CM, Bouwstra JA, Schacht EH, Jungiger HE. In vitro evaluation of mucoadhesive properties of gelatin and some other natural polymer. Int J Pharm,78:43-48, (1992)

11. Henriksen I, Green KL, Smart JD, Smistad G, Karlsen J. Bioadhesive of hydrated gelatin NS: an in vitro and in vivo study. Int J Pharm, 145:231-240, (1996)

12. Chowdary KPR, Rao YS. Design and in vitro and in vivo evaluation of mucoadhesive microcapsule of glipizide for oral controlled release: a technical note. AAPS Pharm Sci Tech,4: E 39, (2003) 
13. Thanoo BC, Sunny MC, Jayakrishnan A. Cross-linked chitosan microspheres: Preparation and evaluation as a matrix for the controlled release of pharmaceuticals. J Pharm Pharmacol, 44:283-286, (1992)

14. Hari PR,Chandy T,Sharma CP.Chitosan / calcium alginate microcapsules for intestinal delivery of Nitrofurantoin. J Microcapsules, 13:319-329.

15. Liu LS, Liu SQ, Ng SY, Froix M,Heller J. Controlled release of interleukin 2 for tumour immunotherapy using alginate/ Gelatin porous microspheres. J Control release,43:65-74(1997)

16. Patel JK, Bodar MS, Amin AF, Patel MM. Formulation and optimization of mucoadhesive microspheres of Metoclopromaide. Int J Pharm Sci, 66:300-305, (2005)

17. Dubey RR, Parikh RH. Two -stage optimization process for formulation of Chitosan microspheres. AAPS Pharm Sci Tech,5: E5, (2004)

18. Longer M.A, Ching HS \& Robinson J.R. Bioadhesive polymers as platforms for oral controlled drug delivery III: Oral delivery of Chlorothiazide using a Bioadhesive polymer. J Pharm Sci. 74:406-11, (1985)

19. Muniyandy Saravanan, Kesavan Bhaskar, Gomathinayagam Maharajan, Kalathil Sadasivan Pillai. Development of Gelatin Microspheres loaded with Diclofenac sodium for intra articular administration. J Drug Target ,19(2):96-103, (2011)
20. Desai KG, Park HJ. Study of gamma-irradiation effects on Chitosan Microparticles. Drug delivery,13:39-50, (2006)

21. Shabaraya AR, Narayanacharyulu R. Design and evaluation of Chitosan microspheres of Metoprolol tartrate for sustained release. Indian J Pharm Sci 65(3):250-52, (2003)

22. Phutane $P$, Shidhaye $S$, Lolitkar $\mathrm{V}$, Ghule $\mathrm{V}$ et al. In vitro evaluation of novel sustained microspheres of gilipizide prepared by the emulsion diffusionevaporation method. J Young Pharma.2(1):35-41, (2010)

23. Ramabargavi JL, Pochaiah B, Mehar CP, Sai Harikrishna $M C$ et al. Formulation and in vitro evaluation of gastro retentive floating tablets of Glipizide. J Chem. Pharm. Res. 5(2):82-96, (2013)

24. Jain AK, Jain CP, Tanwar YS, Naruka PS. Formulation, characterization and In vitro evaluation of floating microspheres of Famotidine as a gastro retentive dosage form. AJPS 3(3):222-226, (2009)

25. Jayavandhan K Patel, Rakesh P Patel, Avani F Amin, Madhathi $M$ Patel. Formulation and evaluation of mucoadhesive glipizide microspheres. AAPS Pharm Sci Tech,6(1): E49-55, (2005)

26. Halder A, Sa B. Preparation and in vitro evaluation of polystyrene-coated Diltiazem-resin complex by Oilin- water Emulsion solvent evaporation method. AAPS Pharm Sci Tech, 7(2):1-8, (2006). 